\title{
Analyzing restrictive elements of leisure time physical activities (Gumushane University sample)
}

\author{
Yeşim Songün ${ }^{\text {a }}$, Ahmet Yılmaz Albayrak ${ }^{1}$, Dursun Katkat $^{2}$, Serdar Bayrakdaroğlu ${ }^{1}$, and \\ Ibrahim $\mathrm{Can}^{1}$ \\ ${ }^{1}$ Gümüşhane University, Physical Education and Sport College, Gümüşhane/Turkey \\ ${ }^{2}$ Mersin University, Physical Education and Sport College, Mersin/Turkey
}

\begin{abstract}
The purpose of this study is to determine the causes which restrict joining leisure physical activities of academic and administrative staff working at University of Gumushane. 236 academic and 169 administrative, in total 405 person participated voluntarily in the study. Leisure time Physical Activity restrictor developed by Öcal (2012), were used as data collector. Descriptive statistics, T-test (Independent Sample Test) and Tukey test from multiple comparison tests were used in study. Consequently, it can be suggested that physical activities of Academic and Administrative Staff at Gumushane University, were influenced by gender, age, marital status, personal status and sport status.
\end{abstract}

\section{Introduction}

Coping with stress occurred by energy expenditure is very significant for keeping the psychological balance of people who keep in step with intensive work program. For this reason, creating leisure time opportunities in separate time intervals to individuals have been institutionalized. In contemporary societies, as working and life conditions keep an individual away from public is quite negative determinant with regard to production $[1,2]$. As a result of many studies, although physical activity habit show that it plays an important role for health and increasing life quality, technology rapidly developing, caused to decrease of physical activity in errands $[3,4]$. In order to make up this negativeness, an individual should refresh himself/herself by creating leisure time opportunities [1,2].

Leisure time is defined as remaining period of time after fulfilling practical needs of life [5]. Leisure time activities, can be defined as participation actions like resting, entertainment, improving knowledge and skills, voluntary participation to society life which an individual can participate with his/her own will after fulfilling professional, familial, and social assignments [6].

\footnotetext{
a Corresponding author: yesimsongun@hotmail.com
} 
Physical activity is effective in preventing postural disorders, getting rid of loneliness by providing social cohesion, protecting muscle joints and tissues, increasing protective and preventive effects against heart and coronary diseases, decreasing tensions, reaching respiratory and circulatory system to highest level, slowing down organic aging, preventing obesity and protecting body against diseases [7].

For this reason, the importance of physical activity for a health life, have been acknowledged by people day by day. In parallel with this, for sustainable active life; it is required to research the causes physical activity and extinguish these causes restricting physical activity. Studies in recent years towards physical activity restrictors will increase participation in these activities with regard to quality and quantity [8]. As a matter of fact, we come across with some studies related to leisure time physical activity restrictors in our country and abroad $[9,10,11,12,13]$.

Governments have been encouraging their people to be active as physical in leisure time activities. Increasing leisure time activities have also triggered active participation in sportive activities [14]. Being active participation rate in physical activities at leisure times is high, arises from individual and social characteristics. Not only Participation in leisure time physical activities is provided easily, but also has a feature to respond to everybody's wishes and pleasures [15].

LTPA's, are classified in three main sections [16]. Low velocity physical activities; include resting, social sharing and relaxing, and also includes activities like hiking, hunting, gardening. Middle velocity activities consist of running, swimming, cycling, cutting trees, collecting woods requiring certain physical activities and physical activity contains weight control and and health life elements. Finally, high velocity physical activities, include jogging and team game activities and it aims to lose weight and detoxication. In addition, this sort of activities, require a optimum level of physical performance. Adults were recommended to join physical activities middle velocity physical activities 30 minutes and five days per week. When considering the benefits of physical activity, more than being a leisure time physical activity, it is obvious that it is a crucial point to be emphasized. Regular physical activity, is directly related to mental and physical health and encouraging regular physical activity has a priority with regard to public health [8].

Universities should be the institutions which aim to raise well-educated individuals useful to society. Because, university students, are the candidates to determine the futures of nations [1]. For this reason, supporting participation of students to physical activities and participation of university staff to physical activity for leading them are quite important. The purpose of this study is to determine the causes which restrict joining leisure physical activities of academic and administrative staff working at University of Gumushane. In this direction; minimizing restrictive effects and developing physical conditions are necessary.

\section{Method}

405 staff, (236) academic, (169) administrative participated in this study. In order to find answers for the purpose of study, the scale of Leisure time Physical Activity restrictors" which was developed by Öcal (2012) were used.

Scale consist of 38 item - 8 sub-dimensions and six fold Likert scale and it measures the elements which restrict participation of participants to physical activity at leisure time. Assessment and perception of individuals related to their own status were examined under "'Physical perception"' sub-dimension, facilities for physical activity in area of residence were under "facility" sub-dimension, budget of individuals for physical activity were under "'Income" sub-dimension, attitudes of close relatives to physical activity were under "Family", perceived individual skills and experiences towards physical activity were under 'Skill Perception", restricted time related to physical activity due to compulsory 
works were under 'Time", making decisions whether doing physical activity or not were under 'Will', and attitudes of individuals or groups for physical activity out of close relatives were under 'Social Environment'.

In order to test reliability of scale, Cronbach Alfa internal consistency coefficient were analysed, and Cronbach Alfa coefficient were calculated as 0,90. Values obtained, demonstrate that LTPA scale is a reliable scale for measuring physical activity restrictors of students to participate in physical activity.

In the analysis of data, descriptive statistics, T-test (Independent Samples T-test) and Tukey test from Multi comparison tests.

\section{Findings}

Table 1. Finding related to gender

\begin{tabular}{ccccccc}
\hline & Gender & $\mathbf{N}$ & $\mathbf{X}$ & $\mathbf{S s}$ & $\mathbf{t}$ & $\mathrm{p}$ \\
\hline Physical perception & Female & 148 & 11,7973 & 5,27471 & \multirow{2}{*}{, 195} &, 84 \\
& Male & 256 & 11,6875 & 5,53244 & & \\
Income & Female & 148 & 14,8716 & 6,85345 & 3,25 &, 00 \\
& Male & 256 & 12,7422 & 6,02380 & & \\
Time/duration & Female & 148 & 11,1149 & 4,06666 &, 862 & .38 \\
& Male & 256 & 10,7539 & 4,04848 & & \\
Family & Female & 148 & 7,7703 & 4,27315 & 1,52 & .12 \\
& Male & 256 & 7,1523 & 3,70218 & & \\
Skill perception & Female & 148 & 9,8851 & 5,18570 & 1,78 &, 07 \\
& Male & 256 & 9,0820 & 3,80880 & & \\
Social environment & Female & 148 & 14,7162 & 5,37447 & 2,04 &, 04 \\
& Male & 256 & 13,5898 & 5,30480 & & \\
Facility & Female & 148 & 26,1824 & 7,98897 & 2,46 &, 01 \\
& Male & 256 & 24,0508 & 8,57181 & & \\
Will & Female & 148 & 26,1824 & 7,98897 & 1,81 &, 07 \\
& Male & 256 & 24,0508 & 8,57181 & 1,81 & \\
\hline
\end{tabular}

When examining Table 1, physical activity restrictor scores of academic and administrative staff; there was no difference seen according to gender in Will sub-dimension, skill perception sub-dimension, Family sub-dimension, Time sub-dimension, physical perception sub-dimension.

But however, physical activity restrictor scores of academic and administrative staff; there were significant differences seen according to gender in facility sub-dimension, social environment sub-dimension, and Income sub-dimension.

In table 2, physical activity restrictor scores of academic and administrative staff; there were significant differences seen according to marital status in time sub-dimension, and in facility sub-dimension.

When examining Table 3, physical activity restrictor scores of academic and administrative staff; there were significant differences seen according to personnel status in Income sub-dimension, skill perception sub-dimension, and Social Environment subdimension.

In Table 4, physical activity restrictor scores of academic and administrative staff; there were significant differences seen according to their status about participation in both professional and amateur sports before they start working at Gümüşhane University in Family sub-dimension ( $\mathrm{t} 403=3.70, \mathrm{p}<05)$, in Will sub-dimension.

When examining Table 5, physical activity restrictor scores of academic and administrative staff; there were significant differences seen according to personnel status in Income sub-dimension, family sub-dimension, and Skill Perception sub-dimension, social environment and facility. 
Table 2. Findings related to Marital Status

\begin{tabular}{|c|c|c|c|c|c|c|}
\hline & Marital Status & $\mathbf{N}$ & $\mathbf{X}$ & Ss & $t$ & p \\
\hline \multirow[t]{2}{*}{ Physical perception } & Married & 236 & 11,8390 & 5,43506 & \multirow{2}{*}{,49 } & \multirow{2}{*}{,62 } \\
\hline & Single & 169 & 11,5680 & 5,42671 & & \\
\hline \multirow[t]{2}{*}{ Income } & Married & 236 & 13,2712 & 6,14459 & \multirow{2}{*}{,94 } & \multirow{2}{*}{, 34} \\
\hline & Single & 169 & 13,8817 & 6,75931 & & \\
\hline \multirow[t]{2}{*}{ Time/duration } & Married & 236 & 11,4619 & 4,37530 & \multirow{2}{*}{3,48} & \multirow{2}{*}{, 00} \\
\hline & Single & 169 & 10,0592 & 3,40291 & & \\
\hline \multirow[t]{2}{*}{ Family } & Married & 236 & 7,3814 & 3,82137 & \multirow{2}{*}{, 06} & \multirow{2}{*}{94} \\
\hline & Single & 169 & 7,3550 & 4,07862 & & \\
\hline \multirow[t]{2}{*}{ Skill perception } & Married & 236 & 9,2881 & 4,08339 & \multirow{2}{*}{,43 } & \multirow{2}{*}{,66 } \\
\hline & Single & 169 & 9,4793 & 4,75731 & & \\
\hline \multirow[t]{2}{*}{ Social environment } & Married & 236 & 13,8644 & 5,29458 & \multirow{2}{*}{, 52} & \multirow{2}{*}{,60 } \\
\hline & Single & 169 & 14,1479 & 5,46052 & & \\
\hline \multirow[t]{2}{*}{ Facility } & Married & 236 & 23,8644 & 8,53370 & \multirow{2}{*}{2,65} & \multirow{2}{*}{, 00} \\
\hline & Single & 169 & 26,1065 & 8,12846 & & \\
\hline \multirow[t]{2}{*}{ Will } & Married & 236 & 11,2542 & 4,50661 & \multirow{2}{*}{,02 } & \multirow{2}{*}{,98 } \\
\hline & Single & 169 & 11,2426 & 4,75160 & & \\
\hline
\end{tabular}

Table 3. Findings related to Personnel Status

\begin{tabular}{|c|c|c|c|c|c|c|}
\hline & Personnel & $\mathbf{N}$ & $\mathbf{X}$ & Ss & $\mathbf{t}$ & $\mathbf{P}$ \\
\hline \multirow{2}{*}{ Physical perception } & Academic & 236 & 11,4703 & 5,48064 & \multirow[b]{2}{*}{1,12} & \multirow[b]{2}{*}{,26 } \\
\hline & Administrative & 169 & 12,0828 & 5,34569 & & \\
\hline \multirow[t]{2}{*}{ Income } & Academic & 236 & 12,5424 & 6,03697 & \multirow[b]{2}{*}{3,70} & \multirow[b]{2}{*}{, 00} \\
\hline & Administrative & 169 & 14,8994 & 6,66962 & & \\
\hline \multirow[t]{2}{*}{ Time/duration } & Academic & 236 & 11,1822 & 4,02924 & \multirow{2}{*}{1,79} & \multirow{2}{*}{, 07} \\
\hline & Administrative & 169 & 10,4497 & 4,06042 & & \\
\hline \multirow[t]{2}{*}{ Family } & Academic & 236 & 7,2669 & 3,88679 & \multirow{2}{*}{, 62} & \multirow{2}{*}{, 53} \\
\hline & Administrative & 169 & 7,5148 & 3,98674 & & \\
\hline \multirow{2}{*}{ Skill perception } & Academic & 236 & 8,8347 & 4,19653 & \multirow{2}{*}{2,97} & \multirow{2}{*}{, 00} \\
\hline & Administrative & 169 & 10,1124 & 4,51510 & & \\
\hline \multirow{2}{*}{ Social environment } & Academic & 236 & 13,5085 & 5,52556 & \multirow{2}{*}{2,11} & \multirow{2}{*}{, 03} \\
\hline & Administrative & 169 & 14,6450 & 5,06076 & & \\
\hline \multirow{2}{*}{ Facility } & Academic & 236 & 24,3263 & 8,52378 & \multirow{2}{*}{1,33} & \multirow{2}{*}{, 18} \\
\hline & Administrative & 169 & 25,4615 & 8,27647 & & \\
\hline \multirow{2}{*}{ Will } & Academic & 236 & 10,9788 & 4,48586 & \multirow{2}{*}{1,39} & \multirow{2}{*}{, 16} \\
\hline & Administrative & 169 & 11,6272 & 4,75314 & & \\
\hline
\end{tabular}

Table 4. Findings related to Participation in Amateur Sports

\begin{tabular}{|c|c|c|c|c|c|c|}
\hline & Sport & $\mathbf{N}$ & $\mathbf{X}$ & Ss & $\mathbf{t}$ & $\mathbf{p}$ \\
\hline \multirow{2}{*}{ Physical perception } & Yes & 194 & 11,3144 & 5,49131 & \multirow{2}{*}{1,46} & \multirow{2}{*}{, 14} \\
\hline & No & 211 & 12,1043 & 5,35133 & & \\
\hline \multirow{2}{*}{ Income } & Yes & 194 & 13,0515 & 6,54339 & \multirow{2}{*}{1,43} & \multirow{2}{*}{, 15} \\
\hline & No & 211 & 13,9621 & 6,26316 & & \\
\hline \multirow{2}{*}{ Time/duration } & Yes & 194 & 10,7577 & 4,32106 & \multirow{2}{*}{, 56} & \multirow{2}{*}{,57 } \\
\hline & No & 211 & 10,9858 & 3,79784 & & \\
\hline \multirow{2}{*}{ Family } & Yes & 194 & 7,9536 & 4,60152 & \multirow{2}{*}{2,89} & \multirow{2}{*}{, 00} \\
\hline & No & 211 & 6,8341 & 3,09623 & & \\
\hline \multirow{2}{*}{ Skill perception } & Yes & 194 & 9,2113 & 4,54929 & \multirow{2}{*}{,69 } & \multirow{2}{*}{,49 } \\
\hline & No & 211 & 9,5118 & 4,20924 & & \\
\hline \multirow{2}{*}{ Social environment } & Yes & 194 & 13,7938 & 5,61906 & \multirow{2}{*}{,68 } & \multirow{2}{*}{,49 } \\
\hline & No & 211 & 14,1564 & 5,11666 & & \\
\hline \multirow{2}{*}{ Facility } & Yes & 194 & 24,5928 & 8,61521 & \multirow{2}{*}{, 47} & \multirow{2}{*}{,63 } \\
\hline & No & 211 & 24,9905 & 8,27158 & & \\
\hline \multirow{2}{*}{ Will } & Yes & 194 & 10,7474 & 4,61728 & \multirow{2}{*}{2,11} & \multirow{2}{*}{, 03} \\
\hline & No & 211 & 11,7109 & 4,55515 & & \\
\hline
\end{tabular}


Table 5. Finding Related to Age Variable

\begin{tabular}{|c|c|c|c|c|c|c|c|}
\hline & Age & $\mathbf{N}$ & $\mathbf{X}$ & Ss & $\mathbf{t}$ & $\mathbf{p}$ & $\begin{array}{l}\text { Significant } \\
\text { Difference }\end{array}$ \\
\hline \multirow{4}{*}{ Physical perception } & $21-30$ & 149 & 11,1409 & 5,16749 & \multirow{5}{*}{1,28} & \multirow{5}{*}{, 27} & \multirow{13}{*}{$\mathrm{A}>\mathrm{C}$} \\
\hline & $31-40$ & 190 & 11,8684 & 5,54906 & & & \\
\hline & $41-50$ & 56 & 12,5357 & 5,89596 & & & \\
\hline & $51-60$ & 10 & 13,2000 & 3,32666 & & & \\
\hline \multirow{4}{*}{ Income } & $21-30$ & 149 & 14,5973 & 7,08912 & & & \\
\hline & $31-40$ & 190 & 13,1737 & 5,73178 & \multirow{3}{*}{4,77} & \multirow{3}{*}{, 00} & \\
\hline & $41-50$ & 56 & 11,3036 & 5,83382 & & & \\
\hline & $51-60$ & 10 & 16,7000 & 7,40945 & & & \\
\hline \multirow{4}{*}{ Time } & $21-30$ & 149 & 10,6510 & 3,96254 & \multirow{5}{*}{1,11} & \multirow{5}{*}{, 31} & \\
\hline & $31-40$ & 190 & 10,9632 & 3,89053 & & & \\
\hline & $41-50$ & 56 & 10,7857 & 4,76622 & & & \\
\hline & $51-60$ & 10 & 13,1000 & 4,01248 & & & \\
\hline \multirow{4}{*}{ Family } & $21-30$ & 149 & 7,1678 & 4,04268 & & & \\
\hline & $31-40$ & 190 & 7,4368 & 3,55214 & \multirow{3}{*}{6,54} & \multirow{3}{*}{, 00} & \multirow{3}{*}{$\begin{array}{c}\mathrm{D}>\mathrm{A}, \mathrm{D}>\mathrm{B}, \\
\mathrm{D}>\mathrm{C}\end{array}$} \\
\hline & $41-50$ & 56 & 6,7679 & 3,50579 & & & \\
\hline & $51-60$ & 10 & 12,5000 & 7,12195 & & & \\
\hline \multirow{4}{*}{ Skill perception } & $21-30$ & 149 & 9,8658 & 4,88469 & \multirow{5}{*}{2,88} & \multirow{5}{*}{, 03} & \multirow{5}{*}{$\mathrm{D}>\mathrm{A}$} \\
\hline & $31-40$ & 190 & 8,8895 & 3,99780 & & & \\
\hline & $41-50$ & 56 & 9,1607 & 3,86018 & & & \\
\hline & $51-60$ & 10 & 12,2000 & 4,58984 & & & \\
\hline \multirow{4}{*}{ Social environment } & $21-30$ & 149 & 14,8523 & 5,35670 & & & \\
\hline & $31-40$ & 190 & 13,6947 & 5,21815 & \multirow{3}{*}{3,09} & \multirow{3}{*}{, 02} & \multirow{3}{*}{$\mathrm{A}>\mathrm{C}$} \\
\hline & $41-50$ & 56 & 12,4821 & 5,79652 & & & \\
\hline & $51-60$ & 10 & 14,9000 & 3,34830 & & & \\
\hline \multirow{4}{*}{ Facility } & $21-30$ & 149 & 25,5906 & 7,66178 & \multirow{5}{*}{5,88} & \multirow{5}{*}{, 00} & \multirow{8}{*}{$\mathrm{A}>\mathrm{C}$} \\
\hline & $31-40$ & 190 & 25,5368 & 8,66399 & & & \\
\hline & $41-50$ & 56 & 20,7321 & 8,90044 & & & \\
\hline & $51-60$ & 10 & 21,8000 & 5,41192 & & & \\
\hline \multirow{4}{*}{ Will } & $21-30$ & 149 & 11,0940 & 5,02204 & & & \\
\hline & $31-40$ & 190 & 11,2421 & 4,25065 & \multirow{3}{*}{, 23} & \multirow{3}{*}{, 87} & \\
\hline & $41-50$ & 56 & 11,6964 & 4,85768 & & & \\
\hline & $51-60$ & 10 & 11,2000 & 3,42540 & & & \\
\hline
\end{tabular}

The findings obtained, demonstrate that age has no significant effect on leisure time physical activity restrictors in academic and administrative staff.

\section{Results and Discussion}

When examining Table 1, physical activity restrictor scores of academic and administrative staff; there were no differentiation seen according to gender in Will sub-dimension ( $\mathrm{t} 402=$ $1.81, \mathrm{p}>05)$, skill perception sub-dimension ( $\mathrm{t} 402=1.78, \mathrm{p}>05)$, Family sub-dimension $(\mathrm{t} 402=1,52, \mathrm{p}>05)$, Time sub-dimension $(\mathrm{t} 402=.86, \mathrm{p}>05)$, physical perception subdimension $(\mathrm{t} 402=.19, \mathrm{p}>05)$.

But however, physical activity restrictor scores of academic and administrative staff; there were significant differences seen according to gender in facility sub-dimension ( $\mathrm{t} 402$ $=3.25, \mathrm{p}<05)$, social environment sub-dimension $(\mathrm{t} 402=2,04, \mathrm{p}<05)$, and Income subdimension $(\mathrm{t} 402=3.25, \mathrm{p}<05)$. When looking at differences among averages; it was determined that in Income and Social Environment sub-dimensions, the average score of females were higher than males, however, in Facility sub-dimension, the average score of males were higher than females. 
According to the results of study which Gümüş et al. [13] carried out, They were determined that gender differences of participants, created a significant difference in leisure time physical activity restrictors. According to the study, leisure time physical activity restrictors scale scores $(2,44 \pm 0,87)$ of female students, seen to be higher than leisure time physical activity restrictors scale scores $(2,20 \pm 0,91)$ of male students. Apart from this, In study of Demir and Demir [17], there were similar results parallel with out study. Culp [18], emphasized that gender has an important effect in social life and also is a factor which restricts leisure time activities of individuals. Handerson et al. [19], suggested that the situations which males and females come across in social life are different and depending on this, their leisure time activities show differences.

When examining Table 2, physical activity restrictor scores of academic and administrative staff; there were no differentiation seen according to marital status in Will sub-dimension ( $\mathrm{t} 403=.02, \mathrm{p}>05)$, social environment sub-dimension $(\mathrm{t} 403=.52, \mathrm{p}>05)$, Skill-perception sub-dimension ( $\mathrm{t} 403=.43, \mathrm{p}>05)$, Family sub-dimension ( $\mathrm{t} 403=, 06$, $\mathrm{p}>05)$, Income sub-dimension $(\mathrm{t} 403=.94, \mathrm{p}>05)$ and physical-perception sub-dimension $(\mathrm{t} 403=.49, \mathrm{p}>05)$.

But however, physical activity restrictor scores of academic and administrative staff; there were significant differences seen according to marital status in time sub-dimension $(\mathrm{t} 403=3.48, \mathrm{p}<05)$, and in facility sub-dimension $(\mathrm{t} 403=2.65, \mathrm{p}<05)$.

When looking at differences among averages; it can be suggested that in Income and Time sub-dimensions, and facility sub-dimension, the average score of married individuals were higher than single ones.

When examining Table 3, physical activity restrictor scores of academic and administrative staff; there were no differentiation seen according to personnel status in physical perception sub-dimension ( $\mathrm{t} 403=1,12, \mathrm{p}>05)$, family sub-dimension ( $\mathrm{t} 403=, 62$, $\mathrm{p}>05)$, Facility sub-dimension ( $\mathrm{t} 403=1,33, \mathrm{p}>05)$, Will sub-dimension $(\mathrm{t} 403=1.39, \mathrm{p}>05)$.

But however, physical activity restrictor scores of academic and administrative staff; there were significant differences seen according to personnel status in Income subdimension $(\mathrm{t} 403=3.70, \mathrm{p}<05)$, skill perception sub-dimension $(\mathrm{t} 403=2.97, \mathrm{p}<05)$, and Social Environment sub-dimension $(\mathrm{t} 403=2.11, \mathrm{p}<05)$. When looking at differences among averages; it was determined that in Income and Skill Perception sub-dimensions, the average score of administrative staff were higher than academic staff, however, in Social Environment sub-dimension, the average score of academic staff were higher than administrative staff.

When examining Table 4, physical activity restrictor scores of academic and administrative staff; there were no differentiation seen according to participation in amateur sports status in physical perception sub-dimension $(\mathrm{t} 403=1,46, \mathrm{p}>05)$, in income subdimension $(\mathrm{t} 403=1043, \mathrm{p}>05)$, Time sub-dimension $(\mathrm{t} 403=, 56, \mathrm{p}>05)$, skill perception sub-dimension ( $\mathrm{t} 403=, 69, \mathrm{p}>05)$, social environment sub-dimension $(\mathrm{t} 403=.47, \mathrm{p}>05)$, facility sub-dimension ( $\mathrm{t} 403=1.39, \mathrm{p}>05)$.

However, physical activity restrictor scores of academic and administrative staff; there were significant differences seen according to their status about participation in both professional and amateur sports before they start working at Gümüşhane University in Family sub-dimension $(\mathrm{t} 403=3.70, \mathrm{p}<05)$, in Will sub-dimension $(\mathrm{t} 403=2.97, \mathrm{p}<05)$. When looking at differences among averages; it was determined that in Family and Will sub-dimensions, the average score of staff who participated in sports before were higher than staff who did not participate in sports.

When examining Table 5, physical activity restrictor scores of academic and administrative staff; there were no differentiation seen according to their age variables in physical perception sub-dimension $(\mathrm{F} 401=1,28, \mathrm{p}>.05)$, time sub-dimension $(\mathrm{F} 401=1,19$, $\mathrm{p}>$.05) Will sub-dimension $(\mathrm{F} 401=, 232, \mathrm{p}>.05)$. 
However, physical activity restrictor scores of academic and administrative staff; there were significant differences seen according to personnel status in Income sub-dimension $(\mathrm{F} 401=4,77, \mathrm{p}<.05)$, family sub-dimension (F401=6,54, $\mathrm{p}<.05)$, and Skill Perception subdimension $(\mathrm{F} 401=2,88, \mathrm{p}<.05)$,

In order to determine the differences among averages, Tukey test from Multi Comparison tests were used, and it was found that scores of individuals who are between 21-30 ages, were significantly higher than scores of individuals who are between 41-50 ages.

In skill perception sub-dimension, average scores of individuals who are 51-60 were higher than individuals who are 21-30.

In Family perception sub-dimension, average scores of individuals who are 51-60 were significantly higher than individuals who are 31-40 and 41-50.

Data obtained, demonstrate that age has no significant effect on leisure time physical activity restrictors in academic and administrative staff.

The findings obtained, demonstrate that age has no significant effect on leisure time physical activity restrictors in academic and administrative staff. It can be seen that the study of Arabacı and Çankaya supports this result [7].

As a result, leisure time physical activity restrictors of university staff show difference with regard to gender, marital status, personal status, participation in amateur sports, and age variables. When looking at the rates of categories; the groups which were exposed to leisure time physical activity restrictors more, as follows; females more than males, married individuals more than single individuals, administrative staff more than academic staff, and staff participated in sports before more than staff who did not participate in sports before.

First of all the causes which hinder the participation in physical activity, should be determined and extinguished in order to encourage physical activity against obesity.

In this regard, for the further researchers; it is recommended to study groups whose leisure time physical activity restrictors are higher and offer a solution for them and it is also recommended to make researches towards extinguishing the restrictors of leisure time physical activity and encouraging physical activities.

\section{References}

1. S. Mansuroğlu, Mediterr Agric Sci (MAS) 15 (2), 53-62, (2002)

2. Önsoy, C., Osmaniye İskenderun Kıyı Kesiminde Ekolojik Planlama İlkelerine Uygun Alan Kullanımının Araştırılması. Ç.Ü. Fen Bilimleri Enstitüsü, Peyzaj Mimarlığı Anabilim Dalı, Doktora Tezi (Basılmamış), Adana, 236 s. (1984)

3. Ball K, Owen N, Salmon J, Bauman A, Gore CJ. Association of physical activity with body weight and fat in men and women. International Journal of Obesity, 25, 914-919. (2001).

4. Haskell WL. Physical activity, sport, and health: Toward the next century. Research Quarterly For Exercise and Sport, 67 (3), 37-47 (1996)

5. Torkildsen G. Recreation and leisure management. 5th Ed. London and New York: Routledge, Taylor and Francis Group (2005)

6. Tütüncü Ö., Aydın İ., Küçükusta D., Avcı N., Taş İ., Üniversite Öğrencilerinin Rekreasyon Faaliyetlerine Katılımını Etkileyen Unsurların Analizi Spor Bilimleri Dergisi Hacettepe J. of Sport Sciences 22 (2), 69-83 (2011)

7. Arabacı R., Çankaya C, Beden Eğitimi Öğretmenlerinin Fiziksel Aktivite Düzeylerinin Araştırılması Eğitim Fakültesi Dergisi XX (1), 1-15 (2007)

8. Öcal K. Ölçek Geliştirme: Serbest Zaman Fiziksel Aktivite Kısıtlayıcıları (SZFA-K). Spor Bilimleri Dergisi, 23 (2), 50-60 (2012) 
9. Güngörmüş H.A. Özel Sağllk Spor Merkezlerinden Hizmet Alan Bireyleri Rekreasyonel Egzersize Güdüleyen Faktörler. Gazi Üniversitesi. Sağlık Bilimleri Enstitüsü, Doktora Tezi (2007)

10. Müderrislioğlu H, Kutay EL, Örnekci Eșen S Kırsal Rekreasyonel Faaliyetlerde Kısitlayıcılar. Tarım Bilimleri Dergisi; 11 (1), 40-44 (2005)

11. Gürbüz B Kentsel Yaşam Sürecinde Rekreasyonel Katılım Sorunları. Gazi Üniversitesi. Sağllk Bilimleri Enstitüsü, Doktora Tezi (2006)

12. Kim B, Heo J, Chun S, Lee Y Construction and Inital Validation of the Leisure Facilitatör Scale. Leisure/Loisir; 34 (4), 391-405 (2011)

13. Gümüş H., Işık Ö., Karakullukçu Ö., Yıldırım İ., Ortaöğretim Kurumlarında Serbest Zaman Fiziksel Aktivite Kisitlayıc1ları, Special Issue on the Proceedings of the 3rd ISCS Conference SI(1): 814-825, (2014)

14. Coalter F The Social Benefits of Sports: An Overview to Inform the Community Plannin Process. Sportscotland Research Raport no.98 (2005)

15. Ramazanoğlu F, Altıngül O, Özer A Sportif Açıdan Rekreasyon Etkinliklerinin Değerlendirilmesi. Doğu Anadolu Bölgesi Araștırmaları Dergisi; s.176 (2004)

16. Laaksonen DE, Lakka HM, Salonen, JT, Niskanen, LK, Rauramaa, RA, Lakka, TA. Low levels of leisure-time physical activity and cardiorespiratory fitness predict development of the metabolic syndrome. Diabetes Care, 25(9), 1612-1618 (2002)

17. Demir C, Demir N "Bireylerin Boş Zaman Faaliyetlerine Katılmalarını Etkileyen Faktörler İle Cinsiyet Arasındaki İlişki: Lisans Öğrencilerine Yönelik Bir Uygulama", Ege Üniversitesi, İktisadi ve İdari Bilimler Fakültesi, Ege Akademik Bakış Dergisi, 6 (1) Ocak,36-48 (2006)

18. Culp, RH "Adolescent Girls and Outdoor Recreation: A Case Study Examining Constraints and Effective Programming." Journal of Leisure Research, 30 (3), 356-379 (1998)

19. Henderson KA, Bialeschki MD, Shaw SM, Freysinger VJ Both Gains and Gaps: Feminist Perspectives on Women's Leisure. State College, PA: Venture Publishing, Inc (1996) 